EOMmUn Communication et organisation

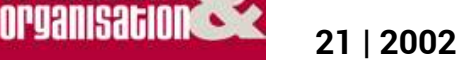

Espace et communication dans les organisations

\title{
Espace et communication
}

Marie-Pascale Mignot

\section{OpenEdition}

Journals

Édition électronique

URL : http://journals.openedition.org/communicationorganisation/2622

DOI : 10.4000/communicationorganisation.2622

ISSN : 1775-3546

Éditeur

Presses universitaires de Bordeaux

Édition imprimée

Date de publication : 1 mai 2002

ISSN : 1168-5549

Référence électronique

Marie-Pascale Mignot, «Espace et communication », Communication et organisation [En ligne],

21 | 2002, mis en ligne le 27 mars 2012, consulté le 30 avril 2019. URL : http://

journals.openedition.org/communicationorganisation/2622; DOI : 10.4000/

communicationorganisation.2622

Ce document a été généré automatiquement le 30 avril 2019

(c) Presses universitaires de Bordeaux 


\title{
Espace et communication
}

\author{
Marie-Pascale Mignot
}

1 La question de la relation de l'espace et de la communication est un vaste domaine, assez diversifié et riche en expériences. Elle concerne plusieurs disciplines: psychologie, sociologie, architecture, urbanisme, muséographie, signalétique, sciences de l'information et de la communication, anthropologie, ethnologie et prend comme terrain d'études de nombreux sites. L'échelle mise à notre disposition est large, elle va de l'espace urbain à l'espace domestique mêlant les genres du public et du privé, avec des nuances autour du semi-public et semi-privé ou encore avec des notions nouvelles comme l'espace de groupe ${ }^{1}$ (agence DEGW) ou la plate-forme de compétences dans les organisations.

2 Selon François Lautier, «les entreprises ont très peu conscience de ce qu'est l'espace, mais elles ont sur cet espace un discours, même s'il ne porte pas explicitement sur lui, celui-ci a une réalité, une matérialité ». Encore inexploité dans les organisations, il tend à être utilisé comme ressource de l'organisation et comme enjeu organisationnel. D'après Gustave-Nicolas Fischer, l'espace est porteur de certains types d'échanges communicationnels. C'est une matrice sociale.

3 Le vingt et unième dossier de Communication et Organisation est une approche resserrée qui cerne la relation de l'espace et de la communication dans les organisations. Le bureau (Fischer-Fousse et Monjaret), mais aussi les lieux hospitaliers (Mercier), l'univers industriel (Bouzon et Dorel-Ferré) ou encore les villages d'enfants SOS (Appel) et les friches portuaires (Gardère) sont abordés comme illustrations du propos. Tous ces espaces engendrent des processus de communication.

4 Ariette Bouzon ouvre le dossier avec une expérience innovante et mène une réflexion sur le « rôle dévolu à l'espace et son influence dans une activité de conception de type ingénierie concourante ». Dans les organisations, quel que soit le type d'activités mis en œuvre, l'espace de travail semble ne plus être secondaire. C'est dans le secteur industriel ou dans les secteurs d'activités de l'information (presse) et de la communication-publicité que les innovations spatiales ont permis les décloisonnements dans l'organisation. Mais ne serait-ce pas l'inverse ? Si on peut agir sur l'espace en l'ouvrant ou en le sectorisant selon les besoins ou les intentions managériales au niveau de la conception architecturale ou de l'aménagement, il faut en revanche savoir répondre aux « besoins antagonistes, de 
communication, d'isolement et d'appropriation d'un territoire individuel des personnes ", qui doivent utiliser ces espaces. L'espace peut être considéré comme une ressource «à optimiser pour agir sur la dynamique sociale ». L'opération reste délicate et semble souvent satisfaisante à l'issue de plusieurs adaptations qui ressortent d'« une négociation sociale » ouverte. Avec Jean-Baptiste Godin, concepteur d'une organisation sociale du travail originale, il ne s'agit plus de négociation mais de progrès social. Gracia Dorel-Ferré nous amène sur des lieux industriels, marqués par les réflexions socialistes de l'ère industrielle du XIX ${ }^{e}$ siècle. En effet, le familistère de Guise en Picardie, imaginé par cet industriel progressiste est la concrétisation architecturale d'une utopie et d'une expérience communicationnelle innovante. Il y a une relation évidente entre le contexte spatial et la volonté de développer chez l'ouvrier "le sens de l'entraide et de la collaboration » (Godin). Ainsi son palais social est organisé autour de cours vitrées, espace de distribution ramenant la vie de chaque habitation vers l'intérieur, espace de rencontre en même temps qu'espace de surveillance. La grande bâtisse à l'allure de palais créé un cadre, une scène où se jouent des interactions.

Violaine Appel soulève l'«ambiguïté entre sphères professionnelle et sphère privée, l'univers professionnel débordant sur la vie privée et vice versa » souvent maintenue par l'enjeu managérial que représente l'espace. Travailler chez soi, travailler au bureau, travailler durant les temps de transport, toutes les formes sont possibles, acceptées et pratiquées. Les espaces se transforment, les objets se miniaturisent. Le fabricant de valises Delsey a mis au point en 1999. une valise-bureau : la « valise trolley business » qui a remporté un franc succès auprès des travailleurs nomades et des cadres perpétuellement en déplacement, capables de travailler n'importe où (train, aéroport, rue, jardin, café...). Travailler n'importe où, mais pas n'importe comment: des expérimentations répondent à cette idée et tendent à domestiquer les espaces de travail. La tendance « cosy » selon Philippe Meurice, architecte, répond aux besoins individuels. La collectivisation n'est pas mise de côté avec le partage des locaux, des outils et même la mise à disposition d'équipements (salle de gymnastique, salle de détente, salle de méditation, pressing, garderie d'enfants...) facilitant la vie privée dans l'enceinte du lieu de travail et rendant le lieu de travail « rassurant, exagérément agréable $»^{2}$. Le confort est accru pour l'individu, l'identité collective est exacerbée. La frontière entre sphère professionnelle et sphère privée n'est plus étanche, avec le risque de faire peser de nouvelles formes de contraintes sur l'employé.

6 Anne Monjaret, après un « détour historique » sur la formation des espaces de bureaux de l'habitation à l'immeuble spécialisé, évoque les lieux de travail comme des espaces plutôt banals et poussiéreux. "Il semble que l'aménagement intérieur et la décoration des espaces comme éléments esthétiques ou signes distinctifs, n'ont pas fait forcément partie des priorités des chefs d'entreprises, des responsables administratifs, plus soucieux d'une rationalisation des tâches professionnelles ». Souvent les chercheurs ont observé le peu d'intérêt porté par les entreprises et les organisations, sur les espaces. Trop légers par leur absence ou trop lourds par leurs contraintes, ils constituent pourtant, un enjeu organisationnel. C'est pour le bureau, terrain d'exploration d'Anne Montjaret qu'il s'agit d'« évaluer l'inscription territoriale des salariés au travail, salariés pleinement ou partiellement sédentaires, en utilisant les objets qui l'entourent comme indicateur d'un potentiel ancrage ». Le bureau est considéré comme un espace de vie, un espace habité. 11 suscite des marquages du territoire, des types d'appropriation, une "articulation entre sphère privée et sphère professionnelle, entre travail et hors-travail». Dans la vie 
quotidienne des employés, des attributs du bureau permettent l'expression d'une personnalité, l'assise des communications qui amènent à construire un bien-être, une « forme de stabilité territoriale et morale ». Ces objets ordinaires sont l'espace d'un entredeux: entre l'espace architectural imposé par l'institution et l'espace de travail nécessairement fonctionnel, qui transgressent les règles tout en inventant les nouveaux caractères d'un espace habité et communicationnel.

Gustave-Nicolas Fischer et Chantai Fousse font la différence entre espace architectural comme outil de communication, support d'image et aménagement intérieur comme réseau de relations communicationnelles. Sur ce dernier point, l'analyse des « concepts et formes d'aménagement liés à l'évolution de l'organisation du travail permettent de comprendre les liens existant entre le fait de configurer l'espace de telle ou telle manière et le fait de la considérer comme performant par rapport à la circulation de l'information et de la communication ». Les modèles sont restreints : bureaux paysagers et bureaux cloisonnés, mais les combinaisons sont multiples (semi-cloisonnés). L'espace s'est peu à peu décloisonné et enrichi de variables très intéressantes et collant de plus en plus avec la culture de l'entreprise, ses valeurs et son organisation. Le décloisonnement de l'espace ne facilite pas systématiquement la communication. Entre cloisonnement et décloisonnement, il est nécessaire d'adapter les modalités spatiales en fonction des résultats souhaités : échanges sur le plan professionnel, niveau de concentration et de confidentialité, polyvalence des employés, sectorisation du personnel, compétition... Les conséquences du déménagement d'un service en milieu hospitalier observé par Nathalie Mercier, dans des «locaux plus vastes et segmentés dans lesquels le confort était supérieur à celui des espaces précédents, modifia les conditions de la communication. Elle devint moins spontanée. Le cloisonnement de l'espace s'accompagna d'un cloisonnement des savoirs et d'une tendance marquée à l'individualisme ».

8 L'émergence des nouvelles technologies d'information et de communication, la réduction du temps de travail, la mobilité ont modifié les organisations. L'apport de l'informatique dans les lieux de travail a engendré de nouveaux concepts d'aménagement. GustaveNicolas Fischer et Chantai Fousse ont analysé le projet appelé working lunch qui consistait «à rechercher le type d'environnement le plus adéquat par rapport aux nouveaux outils technologiques». C'est le restaurant qui a été retenu. Dans un tel environnement, l'individu doit jouer entre des espaces plus personnels et des espaces à partager. Le bureau ne répond plus à une logique de surface et d'ergonomie mais à une logique de service. La question «ne doit plus être de savoir où l'on va s'asseoir en arrivant au bureau, mais de savoir quelle tâche on va accomplir» explique Jay Chiat, lors du réaménagement de son agence de publicité new-yorkaise, inspiré de l'univers du salon de thé. Toute tentative d'organisation de l'espace de travail passe par la compréhension des comportements et des effets créés par le contexte environnemental. Un travail fin de diagnostic et d'« évaluation qualitative » doit précéder toute conception d'aménagement d'espaces basée sur une " compréhension globale de nouvelles données qui déterminent l'activité, à savoir notamment les outils technologiques ». C'est en ces termes, que se poseront les relations entre espace et communication au niveau du travail.

9 L'expression de la hiérarchie se lit dans l'espace avec des constantes bien connues et assez démodées: le plus grand bureau pour le directeur, des attributs liés au confort et accordés en fonction de l'échelon. En effet, Nathalie Mercier dans son article, remarque que dans l'évolution des lieux de travail, les aménagements deviennent plus égalitaires et plus fonctionnels. De même, la pratique professionnelle de Philippe Meurice ${ }^{3}$, aménageur 
d'espace de travail s'appuie sur une nouvelle forme de management qui travaille sur « une organisation en objectifs » qui permet de soumettre «l'aménagement non plus à des règles d'ordre et de hiérarchie mais à une volonté de confort, en gommant les rapports conflictuels et en faisant une place plus grande à l'individu. L'homme a repris le dessus par rapport au process. Les contraintes sur l'espace glissent du champ fonctionnel mécanique au champ sociologique axé principalement sur la notion d'échanges ». Il s'agit de changer ou de transformer les lieux dans l'article de Nathalie Mercier pour de meilleurs ou de moins bons échanges relationnels et de comprendre quelles sont les représentations de l'espace avant et après un déménagement. Les contraintes organisationnelles ou de gestion amènent parfois, des transformations de l'espace ou des déménagements de locaux, dont les résultats du point de vue communicationnel diffèrent en fonction des groupes et des catégories de personnel. Changer de lieux, c'est changer de pratiques pour le service de neurochirurgie de l'hôpital de la Pitié Salpétrière à Paris : «on est passé de liens reposants moins sur la hiérarchie que sur la complicité et l'entraide à la création de groupes s'organisant autour de statuts socioprofessionnels ».

Le dossier se termine par l'article d'Elizabeth Gardère qui cherche à tracer le parcours de la requalification des lieux de travail privés en lieux urbains publics. Les notions de mémoire, d'image urbaine, d'urbanisme de communication et de médiation urbaine se mêlent dans le cas du réaménagement des quais de la Garonne à Bordeaux. Le passage fragile d'un lieu portuaire fortement marqué par son étendue (plusieurs kilomètres), ses activités à un lieu festif tout aussi emblématique dans sa médiatisation et son ambition (un grand lieu de rencontre à l'échelle de l'agglomération) met en jeu l'espace et la communication. Avec l'architecte Jean Nouvel, un point de vue sur l'espace de la séduction et pour Georges Pérec une instabilité spatiale font de l'objet architectural, un objet communicant.

11 Après ce bref voyage en terme d'introduction, dans les contributions des auteurs, que nous remercions, nous vous invitons à approfondir la réflexion sur la relation de l'espace et de la communication avec la lecture du dossier 


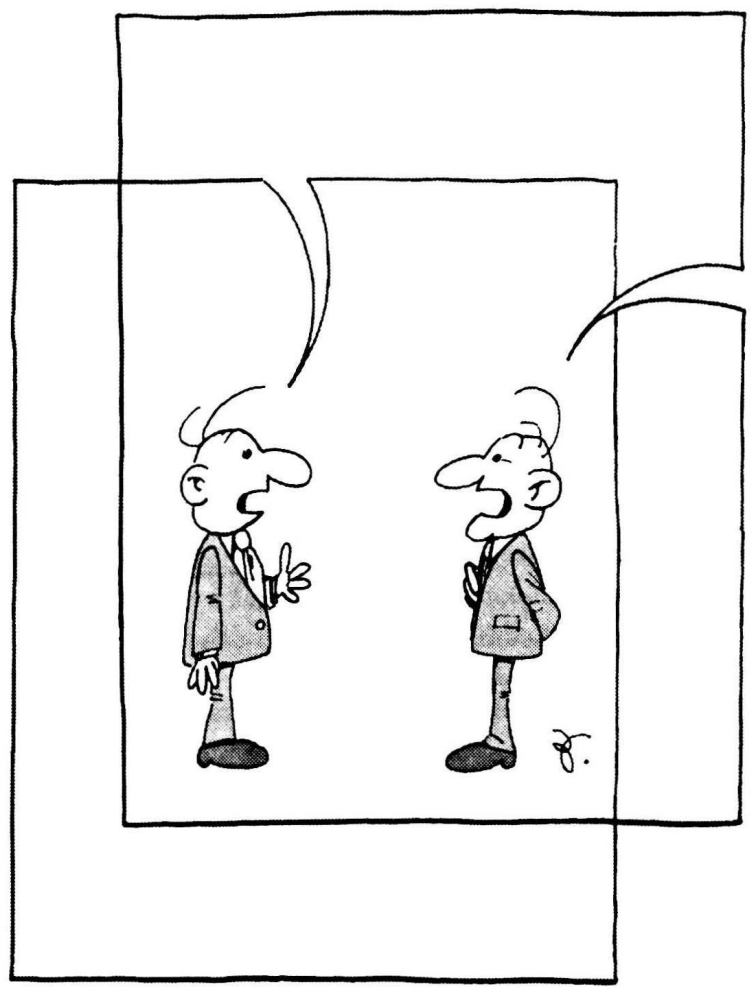

\section{NOTES}

1. Il favorise la synergie et la convivialité de l'équipe. Articulé autour de la circulation secondaire interne, il distribue les différents espaces privés et communs de l'équipe. Il est le lieu d'échanges et le fédérateur de l'équipe.

2. Dana Karine, Sweet bureaux in Revue AMC, spécial tertiaire, édition Le Moniteur - déc. 2001, p. 85

3. Se reporter à la rubrique ENTREVUE 